\title{
Computed tomography Hounsfield units can predict breast cancer metastasis to axillary lymph nodes
}

\author{
Masakazu Urata ${ }^{1}$, Yuko Kijima ${ }^{1 *}$, Munetsugu Hirata ${ }^{1}$, Yoshiaki Shinden ${ }^{1}$, Hideo Arima ${ }^{1}$, Akihiro Nakajo ${ }^{1}$, \\ Chihaya Koriyama², Takaaki Arigami ${ }^{1}$ Yoshikazu Uenosono ${ }^{1}$, Hiroshi Okumura ${ }^{1}$, Kosei Maemura', \\ Sumiya Ishigami ${ }^{1}$, Heiji Yoshinaka ${ }^{1}$ and Shoji Natsugoe ${ }^{1}$
}

\begin{abstract}
Background: Axillary lymph node (ALN) status is an important prognostic factor for breast cancer. We retrospectively used contrast-enhanced computed tomography (CE-CT) to evaluate the presence of ALN, metastasis based on size, shape, and contrasting effects.

Methods: Of 131 consecutive patients who underwent CE-CT followed by surgery for breast cancer between 2005 and 2012 in our institution, 49 were histologically diagnosed with lymph node metastasis. Maximum Hounsfield units (HU) and mean HU were measured in non-contrasting CT (NC-CT) and CE-CT of ALNs.

Results: Of 12 examined measurements, we found significant differences between negative and metastatic ALNs in mean and maximum NC-CT HU, and mean and maximum CE-CT HU $(P<0.05)$. We used a receiver operating curve, to determine cut-off values of four items in which significant differences were observed. The highest accuracy rate was noted for the cut-off value of 54 as maximum NC-CT HU for which sensitivity, specificity, and accuracy rate were 79.6\%, $80.5 \%$ and $80.2 \%$, respectively.
\end{abstract}

Conclusions: $\mathrm{CT} H \mathrm{HU}$ of a patient with breast cancer are absolute values that offer objective disease management data that are not influenced by the screener's ability.

Keywords: Breast cancer, Computed tomography, Hounsfield unit, Lymph node metastasis, Diagnosis,

Axillary lymph node

\section{Background}

Breast cancer is the most common newly diagnosed cancer type and the fifth most common cause of cancer-related death among women in Japan [1]. Status of axillary lymph nodes (ALNs) is the most important predictor of survival [2].

Manual palpation is a well-known method that is used to non-invasively detect ALN metastasis [3]; however, it has low specificity and sensitivity and cannot accurately predict the ALN status [4]. Contrast-enhanced computed tomography (CE-CT) has been used to evaluate ALN

\footnotetext{
* Correspondence: ykijima@m3.kufm.kagoshima-u.ac.jp

'Department of Digestive Surgery, Breast and Thyroid Surgery, Graduate School of Medical and Dental Sciences, Kagoshima University, 8-35-1, Sakuragaoka, Kagoshima 890-8520, Japan

Full list of author information is available at the end of the article
}

metastasis based on size, shape, and contrasting effects [5-10]. Use of whole-body ${ }^{18} \mathrm{~F}$-fluorodeoxy glucosepositron emission tomography (FDG-PET)/CT for breast cancer staging and treatment monitoring has also recently increased due to its ability to detect previously unknown metastases [11-16]. However, its diagnostic accuracy for ALN staging has not yet been established $[16,17]$.

Hounsfield units (HU) are a measure of X-ray attenuation in CT images. This retrospective study evaluated ALN metastasis against $\mathrm{HU}$ to determine optimal cut-off values.

\section{Methods}

\section{Patients}

Between January 2005 and May 2012, 283 consecutive patients with invasive ductal carcinoma (IDC) underwent breast and axillary surgery in our hospital without any 
neoadjuvant systemic therapy such as chemotherapy or endocrine therapy. Of these patients, 131 underwent CE-CT prior to surgery in our hospital using the same system, and were enrolled in this study; the other 152 patients underwent CE-CT using a different system in another hospital. All subjects had undergone mammography, ultrasonography (US), core needle biopsies for their primary breast lesions, bone scintigraphy for preoperative staging. Magnetic resonance imaging, FDGPET, and pathological or cytological examinations for ALNs, depending on the case. Sentinel lymph node (SLN) biopsies were performed on 51 patients with diagnoses of clinical T1N0M0 breast cancer according to the TNM classification [18]. The dye and radioisotope method used to detect SLNs was reported previously [19]. Axillary lymphadenectomy was performed on 27 and 18 patients who were diagnosed with T1-4N1M0 or T2-4N0M0, respectively. Of 51 patients who underwent SLN biopsies, 9 received additional axillary lymphadenectomy due to findings of metastasis by the intraoperative histological examination.

Patient characteristics and the pathological and surgical findings were collected from our database records and individual patient electronic medical records, and was approved by the Institutional Review Board of the Kagoshima University Medical and Dental Hospital. We received informed consent from each study participant and approval for our protocol from the Ethics Committee of Kagoshima University.

Table 1 shows clinicopathological features of the 131 patients enrolled in this study. Their average age was 58.3 years (range: $21-95$ years). One patient was diagnosed with T1mic cancer, 73 with T1, 32 with T2, 7 with T3, and 18 with T4 lesions. Total and partial mastectomies were performed on 74 and 57 patients, respectively. SLN biopsy was performed on 42 patients, SLN biopsy followed by axillary lymphadenectomy on 9 patients, and axillary lymphadenectomy on 80 patients. Of the 131 patients, 49 had lymph node metastasis. All metastases were evaluated as macrometastasis (Table 1 ).

\section{Testing set}

All 95 consecutive patients with IDC who underwent breast and axillary surgery in our hospital between June 2012 and July 2014 with no neoadjuvant therapy underwent CE-CT: 40 before surgery in our hospital using the same system, and the other 55 patients using a different system in another hospital. We enrolled the 40 former patients in this testing set.

\section{CT scanning}

Patients were examined in a supine position with their arms stretched above their heads at the end of inspiration using a CT scanner (Aquilion TM64, Toshiba Medical,
Table 1 Clinical characteristics of the 131 patients

\begin{tabular}{lc}
\hline Variable & \\
\hline Age in years, average (range) & $58.3(21-95)$ \\
Menopause & $41(31.3 \%)$ \\
Pre & $90(68.7 \%)$ \\
Post & \\
Pathological T stage & $1(0.8 \%)$ \\
T1mic & $11(8.4 \%)$ \\
T1a & $21(16.0 \%)$ \\
T1b & $41(31.3 \%)$ \\
T1C & $32(24.5 \%)$ \\
T2 & $7(5.3 \%)$ \\
T3 & $18(13.7 \%)$ \\
T4 & \\
Procedure & $74(56.5 \%)$ \\
Total mastectomy & $57(43.5 \%)$ \\
Partial mastectomy & \\
Lymph node dissection & $42(32.1 \%)$ \\
SNB* without axillary lymphadenectomy & $9(6.9 \%)$ \\
SNB* with axillary lymphadenectomy & $80(61.0 \%)$ \\
Axillary lymphadenectomy & \\
Lymph node metastasis & $49(37.4 \%)$ \\
Negative & $82(62.6 \%)$ \\
\hline
\end{tabular}

*Sentinel lymph node biopsy.

Tokyo, Japan). Scanning parameters included $120 \mathrm{kVp}$, a 0.5 -second tube rotation, $53^{\circ}$ helical pitch, $206-\mathrm{mm}$ table speed, 0.5 second gantry rotation time, and $3-\mathrm{mm}$ thick reconstructed sections. Images were analyzed on SYNAPSE (Fuji Film, Inc., Tokyo, Japan).

We observed 437 ALNs in 131 patients on CT. On some slides, in which both the pectoralis major and minor muscles were detected, we evaluated all lymph nodes without any information on pathological findings. The mean number of evaluated lymph nodes in each patient was 3.34 (range: 2-6). Maximum and mean $\mathrm{HU}$ were measured in non-contrasting $\mathrm{CT}$ (NC-CT) and CE-CT ALNs, contralateral ALNs, the aortic inside arch and the pectoralis major muscle. To measure lymph node $\mathrm{HU}$ with/without internal fat at normal lymph node hila, we traced outlines of lymph nodes by hand to highlight a range of interest (ROI) (Figure 1). To exclude artificial contamination, we excluded these outlines when we evaluated mean $\mathrm{HU}$ values, but included them to determine maximum $\mathrm{HU}$ values. On each lymph node, we also evaluated long- and short-axis diameters, and internal fat density, which indicated absence of central images for bilateral ALNs. The highest value of a patient's several lymph nodes was used as the value for that patient. 


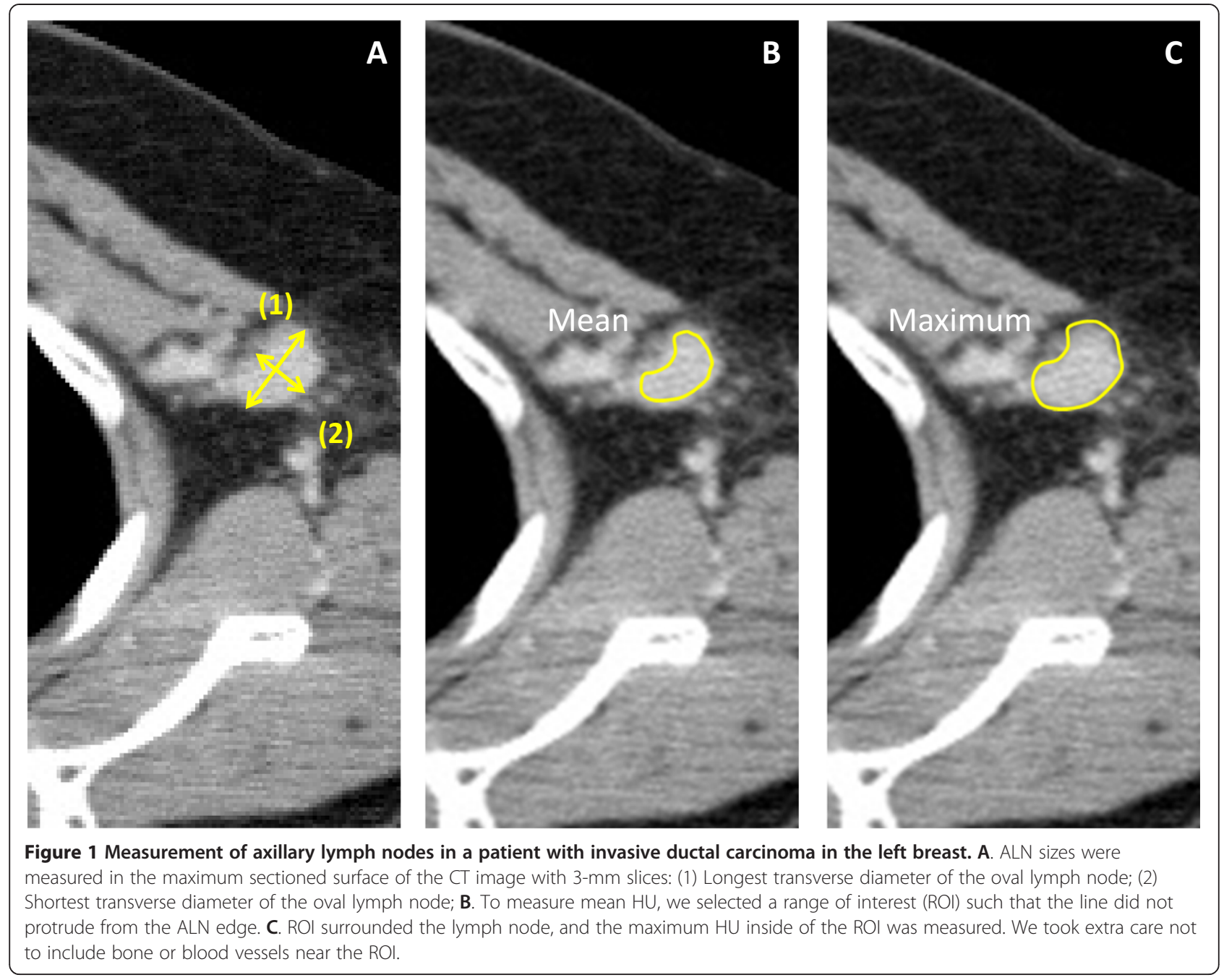

Mean HU, maximum HU, and primary tumor size were simultaneously determined in the same manner.

\section{Pathological evaluation}

All dissected ALNs were cut into single sections and stained with hematoxylin-eosin for analysis by a pathologist. Both micro- and macro-metastasis were considered positive.

\section{Statistical analysis}

Data were evaluated using SPSS Ver.20 software (IBM SPSS, Chicago, IL). Student's $t$-test was used to assess differences between the groups. $P<0.05$ was considered significant. We examined mean $\mathrm{HU}$ and maximum $\mathrm{HU}$ of the ALNs and primary tumor on both NC-CT and CE-CT. We examined differences between metastatic lymph nodes and non-metastatic lymph nodes using 18 measurements, as shown in Table 2. We then used the Youden index of receiver operating characteristic (ROC) curve to determine cut-off values for items in which a significant difference was observed in the $t$-test. The ROC curve was drawn using SPSS Ver.20 software (Figure 2, for an example).

\section{Results}

$\mathrm{HU}$ value and lymph node metastasis

Mean NC-CT HU was $7.83 \pm 22.31$ for non-metastatic lymph nodes and $29.17 \pm 16.37$ for positive nodes. Similarly, maximum $\mathrm{NC}-\mathrm{CT} \mathrm{HU}$, mean $\mathrm{CE}-\mathrm{CT} \mathrm{HU}$, and maximum CE-CT HU were 34.28 $\pm 23.58,60.06 \pm 17.52$ and $52.45 \pm 27.29$ for negative nodes; and $78.09 \pm$ $22.49, \quad 86.95 \pm 27.58$ and $118.63 \pm 23.14$ for positive nodes, respectively (Table 2 ). We also analyzed associations between pathological findings for metastasis and NC-CT and CE-CT HU values for ALNs, primary tumors, and long- and short-axis diameters (Table 2).

Of the 12 values, mean ALN NC-CT HU, maximum ALN NC-CT HU, mean ALN CE-CT HU, maximum ALN CE-CT HU, long- and short-axis ALN diameters, and long- and short-axis diameters of the primary tumor 
Table 2 Comparisons between negative and positive lymph nodes for 12 CT measurements

\begin{tabular}{|c|c|c|c|}
\hline \multirow[b]{2}{*}{ Factors (mean $\pm \mathrm{SD}$ ) } & \multicolumn{3}{|c|}{ Lymph node metastasis } \\
\hline & Negative $(n=82)$ & Positive $(n=49)$ & $P$ \\
\hline Mean NC-CT HU of lymph node & $7.83 \pm 22.31$ & $29.17 \pm 16.37$ & $<0.05$ \\
\hline Maximum NC-CT HU of lymph node & $34.28 \pm 23.58$ & $60.06 \pm 17.52$ & $<0.05$ \\
\hline Mean CE-CT HU of lymph node & $52.45 \pm 27.29$ & $78.09 \pm 22.49$ & $<0.05$ \\
\hline Maximum CE-CT HU of lymph node & $86.95 \pm 27.58$ & $118.63 \pm 23.14$ & $<0.05$ \\
\hline Mean NC-CT HU of primary tumor & $36.09 \pm 18.88$ & $37.28 \pm 16.58$ & 0.89 \\
\hline Maximum NC-CT HU of primary tumor & $69.30 \pm 30.65$ & $78.17 \pm 18.55$ & 0.11 \\
\hline Mean CE-CT HU of primary tumor & $91.92 \pm 28.36$ & $89.03 \pm 31.45$ & 0.39 \\
\hline Maximum CE-CT HU of primary tumor & $135.81 \pm 31.27$ & $148.88 \pm 25.32$ & 0.88 \\
\hline Long-axis diameter of lymph node (mm) & $6.05 \pm 2.32$ & $10.37 \pm 4.75$ & $<0.05$ \\
\hline Short-axis diameter of lymph node (mm) & $4.42 \pm 1.71$ & $7.35 \pm 3.39$ & $<0.05$ \\
\hline Long-axis diameter of primary tumor (mm) & $18.71 \pm 10.14$ & $40.54 \pm 37.94$ & $<0.05$ \\
\hline Short-axis diameter of primary tumor (mm) & $12.99 \pm 7.11$ & $25.44 \pm 33.37$ & $<0.05$ \\
\hline
\end{tabular}

CE-CT: contrast-enhanced computed tomography; HU: Hounsfield unit; NC-CT: non-contrasting computed tomography; SD: standard deviation.

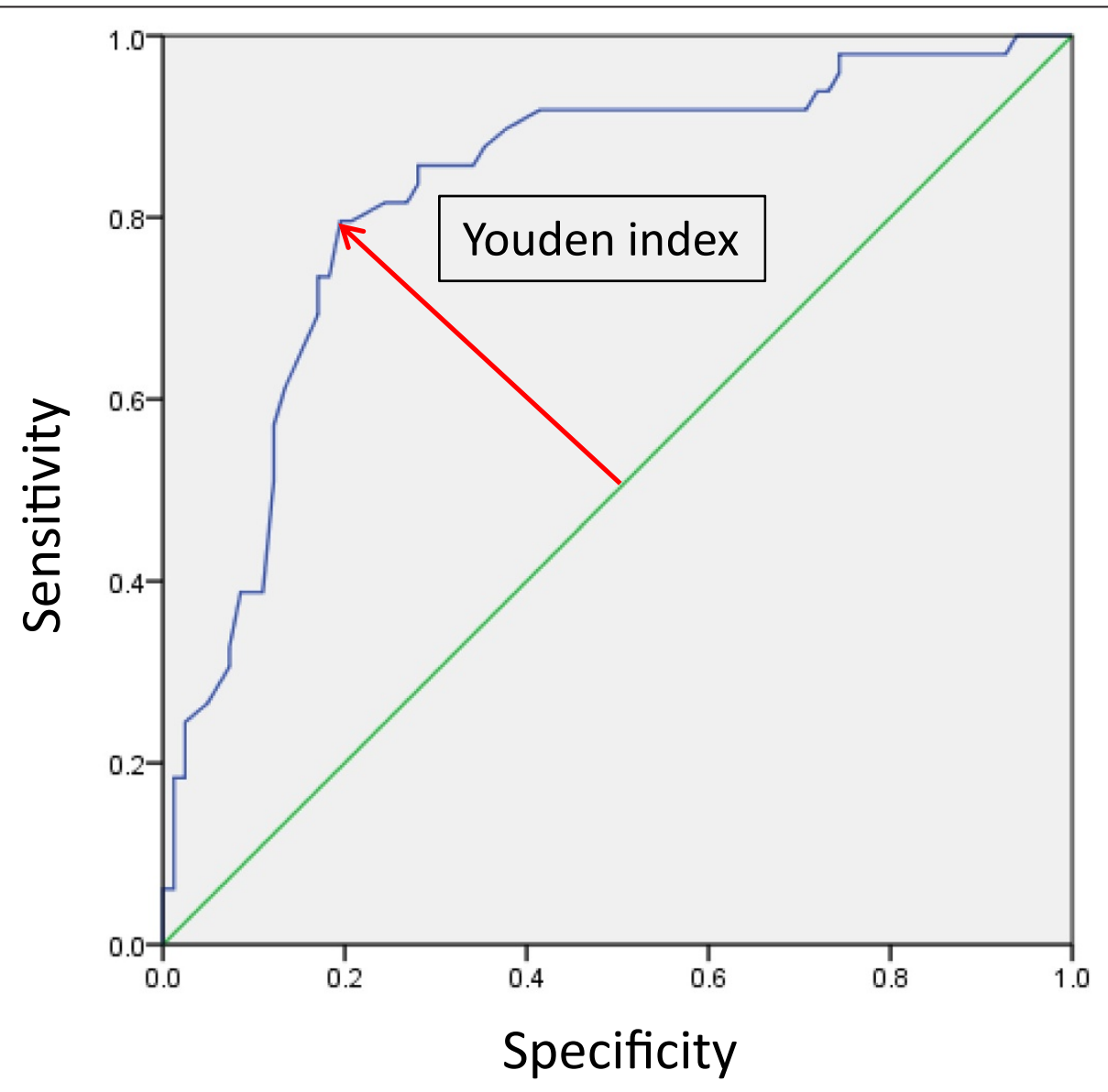

Figure 2 ROC curve of maximum HU of an unenhanced lymph node CT. The AUC of this case was 0.827. We made a ROC curve and found the cut-off value using the Youden index. 
differed significantly for metastatic and negative ALNs; with maximum and mean $\mathrm{HU}$ significantly higher in positive nodes. However, in the primary tumors, none of the CT values correlated with ALN metastasis (Table 2).

Lymph node metastasis and diameters of the lymph node and primary tumor

Long- and short-axis diameters of metastatic lymph nodes were significantly larger than for negative nodes $(P<0.05$, Table 2). Long- and short-axis diameters of primary tumors correlated with lymph node metastasis $(P<0.05$ for both).

\section{Diagnostic accuracy of $\mathrm{HU}$ values in detecting lymph node metastasis}

For the four items with significantly higher $\mathrm{HU}$ values in metastatic lymph nodes-mean and maximum NC-CT $\mathrm{HU}$ values, and mean and maximum $\mathrm{CE}-\mathrm{CT} \mathrm{HU}$ values (Table 3)-we determined HU cut-off values, using the Youden index for ROC curves (Figure 2). The highest accuracy rate was found for maximum ALN NC-CT HU at a cut-off value of 54 (sensitivity: 79.6\%; specificity: 80.5\%; positive predictive value [PPV]: $70.9 \%$; negative predictive value [NPV]: $86.8 \%$; accuracy: $80.2 \%$ ), followed by maximum ALN CE-CT HU at a cut-off value of 103 (sensitivity: 83.7\%; specificity: 72.0\%; PPV: 64.1\%; NPV: 88.1\%; accuracy: 76.3\%), and mean ALN CE-CT HU at a cut-off value of 16 (sensitivity: 83.7\%; specificity: 64.6\%; PPV: $58.6 \%$; NPV: $86.9 \%$; accuracy: $71.8 \%)$. In the same way, we determined size cut-off values and listed sensitivity, specificity, PPV, NPV, and accuracy (Table 4).

\section{Evaluations of testing set}

We used cut-off values for mean and maximum $\mathrm{HU}$ values of both $\mathrm{NC}-\mathrm{CT}$ and $\mathrm{CE}-\mathrm{CT}$ which were derived from 131 cases, to evaluate several lymph nodes in one patient, which we compared with pathologists' findings, as shown in Table 5.

\section{Discussion}

The 10-year survival rate of patients with ALN metastasis depends on the number of involved nodes, and ranges from $30 \%$ for those with $>10$ metastases to $90 \%$ for those with no metastasis [2]. The ALN status is not only important for estimating prognoses, but also for selecting individual treatment regimens [20]. The American College of Surgeons Oncology Group (ACOSOG) Z0011 trial showed that patients randomized to SLN dissection (SLND) alone or to SLND + ALN dissection (ALND), did not significantly differ in local or regional recurrence [21]. The ACOSOGZ0011 enrolled patients who were diagnosed as N0 before randomization, which supports the importance of preoperative ALN evaluation. Our present study could facilitate these evaluations.

The use of CT to assess ALN metastasis has been reported previously [5-10]. Relatively good results were reported for various criteria used to detect lymph node metastasis, such as short-axis ALN diameter, ratio of long- to short-axis ALN diameters, enhancement type, shape, or intra-nodal fat density [10,22-24]. Such methods are useful for detecting ALN metastasis, but may strongly depend on the personal ability of the screener.

Use of US in diagnosing ALN metastasis is also widely reported $[25,26]$. Results of US for non-palpable axillary nodes based on nodal size showed that sensitivity varied between $48.8 \%$ (95\% confidence interval: 39.6-58\%) and $87.1 \%(76.1-94.3 \%)$ and specificity varied between $55.6 \%$ (44.7-66.3\%) and 97.3\% (86.1-99.9\%); for lymph node morphology, sensitivity ranged from $26.4 \%$ (15.3-40.3\%) to $75.9 \%(56.4-89.7 \%)$ and specificity ranged from $88.4 \%$ $(82.1-93.1 \%)$ to $98.1 \%(90.1-99.9 \%)$ [25]. We previously reported that US screening of ALNs was useful both for diagnosing nodal metastasis, and for predicting prognoses. We showed that US sensitivity, specificity and accuracy rates were $69.5 \%, 85.8 \%$ and $79.7 \%$, respectively [26]; however, its ease of use is somewhat offset by the influence of operator's skill and possible subjectivity, whereas measuring CE-CT HU is a simple, easy method that does not depend on the physician's skill.

In a voxel with average linear attenuation coefficient $\mu_{x}$, the corresponding $\mathrm{HU}$ value is given by: $H U=$ $1000 \times\left(\mu_{x}-\mu_{\text {water }}\right) / \mu_{\text {water }}-\mu_{\text {air }}$ where $\mu_{\text {water }}$ and $\mu_{\text {air }}$ are the linear attenuation coefficients of water and air; i.e.,

Table 3 HU measurements that differed significantly in metastatic and negative lymph nodes

\begin{tabular}{|c|c|c|c|c|c|c|}
\hline & $\begin{array}{c}\text { Cutoff value } \\
\text { (HU) }\end{array}$ & $\begin{array}{c}\text { Sensitivity } \\
\text { (\%) }\end{array}$ & $\begin{array}{c}\text { Specificity } \\
(\%)\end{array}$ & $\begin{array}{l}\text { PPV } \\
(\%)\end{array}$ & $\begin{array}{l}\text { NPV } \\
(\%)\end{array}$ & $\begin{array}{c}\text { Accuracy } \\
\text { (\%) }\end{array}$ \\
\hline \multicolumn{7}{|l|}{ Lymph node NC-CT } \\
\hline Mean HU & 16 & 83.7 & 64.6 & 58.6 & 86.9 & 71.8 \\
\hline Maximum & 54 & 79.6 & 80.5 & 70.9 & 86.8 & 80.2 \\
\hline \multicolumn{7}{|l|}{ Lymph node CE-CT } \\
\hline Mean HU & 60 & 85.7 & 58.5 & 55.3 & 87.3 & 68.7 \\
\hline Maximum HU & 103 & 83.7 & 72 & 64.1 & 88.1 & 76.3 \\
\hline
\end{tabular}

CE-CT: contrast-enhanced computed tomography; HU: Hounsfield unit; NC-CT: non-contrasting computed tomography; NPV: negative predictive value; PPV: positive predictive value. 
Table 4 Size measurements that differed significantly in metastatic and negative lymph nodes

\begin{tabular}{|c|c|c|c|c|c|c|}
\hline & $\begin{array}{l}\text { Cut-off value } \\
\qquad(\mathrm{mm})\end{array}$ & $\begin{array}{c}\text { Sensitivity } \\
\text { (\%) }\end{array}$ & $\begin{array}{c}\text { Specificity } \\
\text { (\%) }\end{array}$ & $\begin{array}{l}\text { PPV } \\
\text { (\%) }\end{array}$ & $\begin{array}{l}\text { NPV } \\
(\%)\end{array}$ & $\begin{array}{c}\text { Accuracy } \\
\text { (\%) }\end{array}$ \\
\hline \multicolumn{7}{|l|}{ Lymph node } \\
\hline Long-axis dia & 7.42 & 75.5 & 74.4 & 63.8 & 83.6 & 74.8 \\
\hline Short-axis dia & 4.96 & 81.6 & 73.2 & 64.5 & 87 & 76.3 \\
\hline \multicolumn{7}{|l|}{ Primary tumor } \\
\hline Long-axis dia & 17.08 & 91.7 & 56.1 & 91.7 & 56.1 & 69.2 \\
\hline Short-axis dia & 16.27 & 57.1 & 81.7 & 65.1 & 76.1 & 72.5 \\
\hline
\end{tabular}

Dia: diameter; NPV: negative predictive value; PPV: positive predictive value.

attenuation values expressed in $\mathrm{HU}$ are relative to the attenuation of radiation in water. Positive values represent tissues with attenuation values higher than that of water and negative values represent tissues with lower values. The number 1000 , sometimes called the magnifying value,

Table 5 Testing set results for lymph node metastases; CT values compared with pathological findings

\begin{tabular}{llccc}
\hline Mean NC-CT HU & & \multicolumn{2}{c}{ Pathological findings } & \\
\cline { 3 - 4 } & & Positive & Negative & Total \\
\hline $\mathrm{CT}^{\dagger}$ & Positive & 9 & 9 & 18 \\
& Negative & 1 & 21 & 22 \\
& Total & 10 & 30 & 40
\end{tabular}

Sensitivity 90.0\%, Specificity 70.0\%, PPV 50.0\%, NPV 95.5\%, Accuracy $75.0 \%$

\begin{tabular}{llccc}
\hline Maximum NC-CT HU & & \multicolumn{2}{c}{ Pathological findings } & \\
\cline { 3 - 4 } & & Positive & Negative & Total \\
\hline $\mathrm{CT}^{\dagger}$ & Positive & 10 & 7 & 17 \\
& Negative & 0 & 23 & 23 \\
& Total & 10 & 30 & 40
\end{tabular}

Sensitivity $100.0 \%$, Specificity $76.7 \%$, PPV 58.8\%, NPV 100.0\%, Accuracy $82.5 \%$

\begin{tabular}{llccc}
\hline Mean CE-CT HU & & \multicolumn{2}{c}{ Pathological findings } & \\
\cline { 3 - 4 } & & Positive & Negative & Total \\
\hline $\mathrm{CT}^{\dagger}$ & Positive & 7 & 13 & 20 \\
& Negative & 3 & 17 & 20 \\
& Total & 10 & 30 & 40
\end{tabular}

Sensitivity 70.0\%, Specificity 56.7\%, PPV 35.0\%, NPV 85.0\%, Accuracy $60.0 \%$

\begin{tabular}{llccc}
\hline Maximum CE-CT HU & & \multicolumn{2}{c}{ Pathological findings } & \\
\cline { 3 - 4 } & & Positive & Negative & Total \\
\hline $\mathrm{CT}^{\dagger}$ & Positive & 8 & 12 & 20 \\
& Negative & 2 & 18 & 20 \\
& Total & 10 & 30 & 40
\end{tabular}

Sensitivity $80.0 \%$, Specificity $60.0 \%$, PPV 40.0\%, NPV 90.0\%, Accuracy $65.0 \%$

${ }^{\dagger}$ Diagnosis of metastasis through $\mathrm{CT} \mathrm{HU}$ values.

CE-CT: contrast-enhanced computed tomography; HU: Hounsfield unit; NC-CT: non-contrasting computed tomography; NPV: negative predictive value; PPV: positive predictive value. is incorporated into the above equation to expand the scale sufficiently to provide whole number attenuation values [27]. Mean HU can be influenced by ROI selection, whereas maximal HU does not vary with ROI placement. Although selecting an ROI cannot currently be automated or standardized, it is an easy manual skill that requires no special technique or software. However, neighboring structures (e.g., bone or blood vessels) should be carefully avoided in selecting a ROI, as maximum HU markedly changes when structures other than lymph nodes are included. Further study is needed to clarify the accuracy of segmentation for ROI delineation; such investigation might show how much of a learning curve exists to acquire the stable ability to determine the $\mathrm{HU}$ value by non-experts. We found sensitivity, specificity, PPV, $\mathrm{NPV}$, and accuracy were 79.6\%, 80.5\%, 70.9\%, 86.8\%, and $80.2 \%$, respectively, at a cut-off value of 54 for maximum $\mathrm{NC}-\mathrm{CT} \mathrm{HU}$. As this result was superior to the findings of previous studies $[11,17,25]$, clinical use of this cut-off value is feasible. Furthermore, accuracy rates were $76.3 \%, 71.8 \%$, and $68.7 \%$ for maximum CE-CT $\mathrm{HU}$, mean NC-CT HU, and mean CE-CT HU, respectively. Therefore, the maximum NC-CT HU cutoff appears adequate to estimate ALN metastasis. Our results showed that NC-CT effectively detected metastasis, and is clearly superior to CE-CT and PET-CT, even from the viewpoint of side effects and cost. In any case, we recommend that $\mathrm{HU}$ be measured to evaluate lymph node metastasis when deciding preoperative staging.

The testing set results under blind conditions validated the analysis. The cut-off value for maximum NC-CT HU showed sensitivity, specificity, PPV, NPV, and accuracy of $100.0 \%, 76.7 \%, 58.8 \%, 100.0 \%$, and $82.5 \%$, respectively, which is particularly useful in light of how easily obtainable this measurement is in routine preoperative examinations.

To our knowledge, this is the first study to show that metastatic and negative ALNs differ in their mean and maximum HU values. Metastases that contain tumor cells, vascularization, or immune reactions within lymph nodes may elevate $\mathrm{HU}$ values. Although we did not evaluate the relationship between metastatic area and 
HU values, further studies should be performed to clarify this issue.

This study had three limitations. First, maximum HU may measure artifacts [28], and no conclusive evidence currently shows that maximum $\mathrm{HU}$ can accurately assess ALN metastasis. We also examined $\mathrm{HU}$ in $3-\mathrm{mm} \mathrm{CT}$ slices in this study; however, $\mathrm{HU}$ values on image borders may be inaccurate for small structures such as lymph nodes due to the partial volume effect. Secondly, HU values depend on the $\mathrm{CT}$ machine, imaging conditions, and specifications of the image processing software, which differ in every institution, and may vary due to maintenance or an update. A cut-off value for $\mathrm{HU}$ in detecting ALN metastasis may only apply to selected patients who undergo NC-CT screening in the same institution. Thus, cut-off values should be regularly recalibrated before treating patients. Finally, comparing one-to-one correspondences between resected lymph nodes and CT ALN images in the present retrospective study was difficult. Confirming relationships between preoperative CT HU and intraoperative and postoperative histological evaluations should resolve these problems. We will be able to achieve higher accuracy by measuring several ALNs followed by the selection of one node with the highest $\mathrm{HU}$ value.

\section{Conclusions}

In conclusion, we have shown that measuring the maximum HU is a simple, easy, and useful technique for diagnosing ALN metastasis in breast cancer patients. However, as this was a retrospective study with relatively few patients, our results should be verified by a blinded prospective investigation by several researchers and a larger cohort.

\section{Abbreviations}

ALN: Axillary lymph node; CE-CT: Contrast-enhanced computed tomography; $\mathrm{CT}$ : Computed tomography; FDG: ${ }^{18}$ F-fluorodeoxy glucose; HU: Hounsfield unit; IDC: Invasive ductal carcinoma; NC-CT: Non-contrasting computed tomography; NPV: Negative predictive value; PET: Positron emission tomography; PPV: Positive predictive value; ROC: Receiver operating characteristic; ROI: Range of interest; SLN: Sentinel lymph node.

\section{Competing interests}

The authors declare that they have no competing interests.

\section{Authors' contributions}

$\mathrm{MU}$ is first author of this work. MU, YK, MH and YS designed all the experiments. MU, HA, AN, YU and $\mathrm{HO}$ acquired data. CK and TA analyzed data. $\mathrm{KM}, \mathrm{SI}, \mathrm{HY}$ and $\mathrm{SN}$ were involved in manuscript revision. All authors were involved in drafting the manuscript and all read it and gave their final approval.

\section{Acknowledgements}

We declare no financial relationship or other interests associated with this manuscript, which might be construed as constituting a conflict of interest. We've stated that the material has not been previously published or submitted elsewhere for publication.

\section{Author details}

'Department of Digestive Surgery, Breast and Thyroid Surgery, Graduate School of Medical and Dental Sciences, Kagoshima University, 8-35-1, Sakuragaoka, Kagoshima 890-8520, Japan. ${ }^{2}$ Department of Epidemiology and Preventive Medicine, Graduate School of Medical and Dental Sciences, Kagoshima University, Kagoshima, Japan.

Received: 18 March 2014 Accepted: 26 September 2014

Published: 30 September 2014

\section{References}

1. Matsuda A, Matsuda T, Shibata A, Katanoda K, Sobue T, Nishimoto H. Cancer Incidence and Incidence Rates in Japan in,2007. A Study of 21 Population-based Cancer Registries for the Monitoring of Cancer Incidence in Japan (MCIJ) Project. Jpn J Clin Oncol 2007, 2013(43):328-336.

2. Miller $W R$, Ellis IO, Sainsbury JR, Dixon JM: ABC of breast diseases: Prognostic factors. BMJ 1994, 309:1573-1576.

3. Fisher B, Wolmark N, Bauer M, Redmond C, Gebhardt M: The accuracy of clinical nodal staging and of limited axillary dissection as a determinant of histologic and nodal status in carcinoma of the breast. Surg Gynecol Obstet 1981, 152:765-772.

4. Sacre RA: Clinical evaluation of axillary lymph nodes compared to surgical and pathological findings. Eur J Surg Oncol 1986, 12:169-173.

5. Ogawa Y, Nishioka A, Nishigawa T, Kubota K, Kariya S, Yoshida S, Tanaka Y, Moriki T, Tochika N: Thin-section CT evaluation and pathologic correlation of therapeutic nodes of clinically node-positive breast cancer patients. Oncol Rep 2003, 10:985-989.

6. Hata Y, Ogawa Y, Nishioka A, Inomata T, Yoshida S: Thin section computed tomography in the prone position for detection of axillary lymph node metastasis in breast cancer. Oncol Rep 1998, 5:1403-1406.

7. Yuen S, Sawai K, Ushijima Y, Okuyama C, Yamagami T, Yamada K, Nishimura $\mathrm{T}$ : Evaluation of axillary status in breast cancer. CT-based determination of sentinel lymph node size. Acta Radiol 2002, 43:579-586.

8. Yuen S, Yamada K, Goto M, Sawai K, Nishimura T: CT-based evaluation of axillary sentinel lymph node status in breast cancer: value of added contrast-enhanced study. Acta Radiol 2004, 45:730-737.

9. Miyauchi M, Yamamoto N, Imanaka N, Matsumoto M: Computed tomography for preoperative evaluation of axillary nodal status in breast cancer. Breast Cancer 1999, 6:243-248.

10. Shien T, Akashi-Tanaka S, Yoshida M, Hojo T, Iwamoto E, Miyakawa K, Kinoshita T: Evaluation of axillary status in patients with breast cancer using thin-section CT. Int J Clin Oncol 2008, 13:314-319.

11. Heusner TA, Kuemmel S, Hahn S, Koeninger A, Otterbach F, Hamami ME, Kimmig KR, Forsting M, Bockisch A, Antoch G, Stahl A: Diagnostic value of full-dose FDG PET/CT for axillary lymph node staging in breast cancer patients. Eur J Nucl Med Mol Imaging 2009, 36:1543-1550.

12. Zangheri B, Messa C, Picchio M, Gianolli L, Landoni C, Fazio F: PET/CT and breast cancer. Eur J Nucl Med Mol Imaging 2004, 31:135-142.

13. Lee JH, Rosen EL, Mankoff DA: The Role of Radiotracer Imaging in the Diagnosis and Management of Patients with Breast Cancer: Part 2-Response to Therapy, Other Indications, and Future Directions. J Nucl Med 2009, 50:738-748.

14. Heusner TA, Kuemmel S, Umutlu L, Koeninger A, Freudenberg LS, Hauth EAM, Kimmig KR, Forsting M, Bockisch A, Antoch G: Breast cancer staging in a single session: whole-body PET/CT mammography. J Nucl Med 2008, 49:1215-1222.

15. Wang Y, Yu J, Liu J, Tong Z, Sun X, Yang G: PET-CT in the diagnosis of both primary breast cancer and axillary lymph node metastasis: initial experience. Int J Radiat Oncol Biol Phys 2003, 57:362-3623.

16. Yang WT, Le-Petross HT, Macapinlac H, Carkaci S, Gonzalez-Angulo AM, Dawood S, Resetkova E, Hortobagyi GN, Cristofanilli M: Inflammatory breast cancer: $\mathrm{PET} / \mathrm{CT}, \mathrm{MRI}$, mammography, and sonography findings. Breast Cancer Res Treat 2008, 109:417-426.

17. Ueda S, Tsuda H, Asakawa H, Omata J, Fukatsu K, Kondo N, Kondo T, Hama Y, Tamura K, Ishida J, Abe Y, Mochizuki H: Utility of 18 F-fluoro-deoxyglucose emission tomography/computed tomography fusion imaging (18 F-FDG $\mathrm{PET} / \mathrm{CT}$ ) in combination with ultrasonography for axillary staging in primary breast cancer. BMC Cancer 2008, 8:165.

18. Sobin LH, Hermanek P, Hutter RP: TNM classification of malignant tumors. A comparison between the new (1987) and the old editions. Cancer 1988, 61:2310-2314. 
19. Kijima $Y$, Yohinaka H, Uenosono $Y$, Funasako $Y$, Ehi K, Yanagita S, Arima $H$, Kozono T, Arigami T, Natsugoe S, Aikou T: Intramammary sentinel lymph node in patients with breast cancer: Report of four cases. Surg Today 2008, 38:536-540.

20. Ranaboldo CJ, Mitchel A, Royle GT, Theaker GM, Taylor I: Axillary nodal status in women with screen-detected breast cancer. Eur J Surg Oncol 1993, 19:130-133.

21. Giuliano AE, McCall L, Beitsch P, Whitworth PW, Blumencranz P, Leitch AM, Saha S, Hunt KK, Morrow M, Ballman K: Locoregional recurrence after sentinel lymph node dissection with or without axillary dissection in patients with sentinel lymph node metastases: the American College of Surgeons Oncology Group Z0011 randomized trial. Ann Surg 2010, 252(3):426-432. discussion 432-433.

22. Mizuno $Y$, Satake H, Sawaki A, Sawaki M, Hayashi H, Kobayashi H, Hibi Y, Shibata A, Kikumori T, Imai T: Preoperative Axillary Lymph Node Evaluation by MDCT Imaging. Japanese of Breast Cancer 2006, 21:367-371.

23. Nasu Y, Shikishima H, Miyasaka Y, Nakakubo Y, Ichinokawa K, Kaneko T: A Study of the Assessment of Axillary Lymph Nodes Before Surgery for Breast Cancer Using Multidetector-Row Computed Tomography. Surg Today 2010, 40:1023-1026.

24. Uematsu T, Sano M, Homma K: In Vitro High-Resolution Helical CT of Small Axillary Lymph Nodes in Patients with Breast Cancer: Correlation of CT and Histology. Am J Roentgenol 2001, 176:1069-1074.

25. Alvarez S, Anorbe E, Alcorta P, López F, Alonso I, Cortés J: Role of sonography in the diagnosis of axillary lymph node metastases in breast cancer: a systematic review. Am J Roentgenol 2006, 186:1342-1348.

26. Kijima Y, Yoshinaka H, Hirata M, Mizoguchi T, Ishigami S, Nakajo A, Arima H, Ueno $S$, Natsugoe S: Number of axillary lymph node metastases determined by preoperative ultrasound is related to prognosis in patients with breast cancers. Cancer 2010, 2:20-31.

27. Michel M: In Computed body tomography. Edited by Lee JKT, Sagel SS, Stanley RJ. New York: Raven; 1983:4-5.

28. Mori I, Kazama M: Method for Suppressing Streak Artifacts in CT Resulting from Excessive Noise. Med Imaging Technol 2003, 21:272-276.

doi:10.1186/1471-2407-14-730

Cite this article as: Urata et al:: Computed tomography Hounsfield

units can predict breast cancer metastasis to axillary lymph nodes. BMC Cancer 2014 14:730.

\section{Submit your next manuscript to BioMed Central and take full advantage of:}

- Convenient online submission

- Thorough peer review

- No space constraints or color figure charges

- Immediate publication on acceptance

- Inclusion in PubMed, CAS, Scopus and Google Scholar

- Research which is freely available for redistribution 